\title{
Methanol maser structure and variability
}

\section{Szymczak* A. Bartkiewicz, P. Wolak}

Toruń Centre for Astronomy, Nicolaus Copernicus University, Poland

E-mail: msz@astro.uni.torun.pl, annan@astro.uni.torun.pl, wolakeastro.uni.torun.pl

\begin{abstract}
A sample of massive young stellar objects was mapped at two epochs in the $6.7 \mathrm{GHz}$ methanol maser line using the EVN and monitored with the Torun $32 \mathrm{~m}$ dish over a period of $\sim 14$ months. Preliminary results show variations in the brightness of existing components but no large changes in the overall morphology of emission in the majority of sources during 2-6 years. A level of variability estimated from the single dish data was usually moderate in the most of targets. Remarkably high variability in the flux density and morphology of individual clusters was found in the source G33.641-0.228. In this object an arc-like structure of maser emission likely traces a passage of shock front.
\end{abstract}

10th European VLBI Network Symposium and EVN Users Meeting: VLBI and the new generation of radio arrays

September 20-24, 2010

Manchester, $U K$

\footnotetext{
*Speaker.

${ }^{\dagger}$ This research is supported by the Polish Ministry of Science and Higher Education (grant N N203 386937).
} 


\section{Introduction}

Interferometric studies of $6.7 \mathrm{GHz}$ methanol masers have revealed a wide diversity of morphological structures at sub-arcsecond scales $[1,3,6]$. Single dish measurements of the maser flux in several sources documented a significant level of variability on time-scales of a few days to several years $[2,4]$. In this report we present the preliminary results coming from the EVN and single dish observations. Our purpose was to explore the characteristics of maser variability and examine whether the variations of flux density are related to changes in spot distribution. That should enable us to validate the competing scenarios of methanol maser origin playing in the environments of recent or ongoing high-mass star formation.

\section{Observations}

Full details of the EVN observations and data reduction are described in [1]. Specifically, the observational setup was the same as in the run 1 (see Table 4 in [1]). A typical synthesized beam was $\sim 6 \times 12$ mas and an rms noise level was $\sim 5 \mathrm{mJy} \mathrm{beam}^{-1}$ per channel of $0.09 \mathrm{~km} \mathrm{~s}^{-1}$ width. That survey consists of the first epoch data-set. Second epoch EVN observations of 12 methanol masers from Bartkiewicz et al.'s sample of 31 sources [1] were carried out on 2009 May 29 and 30. The Yebes telescope participated in the observation and the beam was $\sim 6 \times 10$ mas. Those targets were identified using the Torun $32 \mathrm{~m}$ telescope as highly variable at $6.7 \mathrm{GHz}$; i.e. the integrated flux density varied by more than $30 \%$ and amplitude of individual features changed by a factor of more than two on time-scales of $6-7$ years.

The $6.7 \mathrm{GHz}$ flux density was systematically monitored with the $32 \mathrm{~m}$ dish from 2009 June to 2010 September. Each target was observed at intervals of $1-6$ weeks. The spectrometer was configured to have a $4 \mathrm{MHz}$ bandwidth with 4096 spectral channels yielding a channel spacing of $0.04 \mathrm{~km} \mathrm{~s}^{-1}$. The half-power beam width was 5.5. The observations were made in frequency switching mode. The system temperature was typically around $40 \mathrm{~K}$ during our observations. The flux density scale was determined by observations of 3C123 [5] and the sensitivity of the telescope was regularly measured by observing of slightly variable source G32.745-0.076 [2]. A careful inspection revealed that some features in the spectrum of this source were essentially stable within $2-3 \%$ in the observing period. The absolute calibration for the flux density was estimated to be better than $10 \%$. A mean rms noise level was $0.2-0.3 \mathrm{Jy}$ in the spectra.

\section{Results and discussion}

In this paper we focus on the source G33.641-0.228 which was observed at a few occasions in the period of $2001-2008$ with the $32 \mathrm{~m}$ telescope and has been appeared as extremely variable. The maser spots mapped at two epochs with the EVN generally preserve the arc-like distribution (Fig. 1) of length of $\sim 150$ mas but the morphology of individual clusters changed significantly. Two new components appeared in 2009 while two others seen in 2003 disappeared. We note that the emission at velocities higher than $62 \mathrm{~km} \mathrm{~s}^{-1}$ showed the strongest changes in the intensity and structure. Comparison of distribution at the two epochs reveals the largest relative displacement of maser clusters of less than 1.2 mas that corresponds to the relative tangential motion with a velocity 


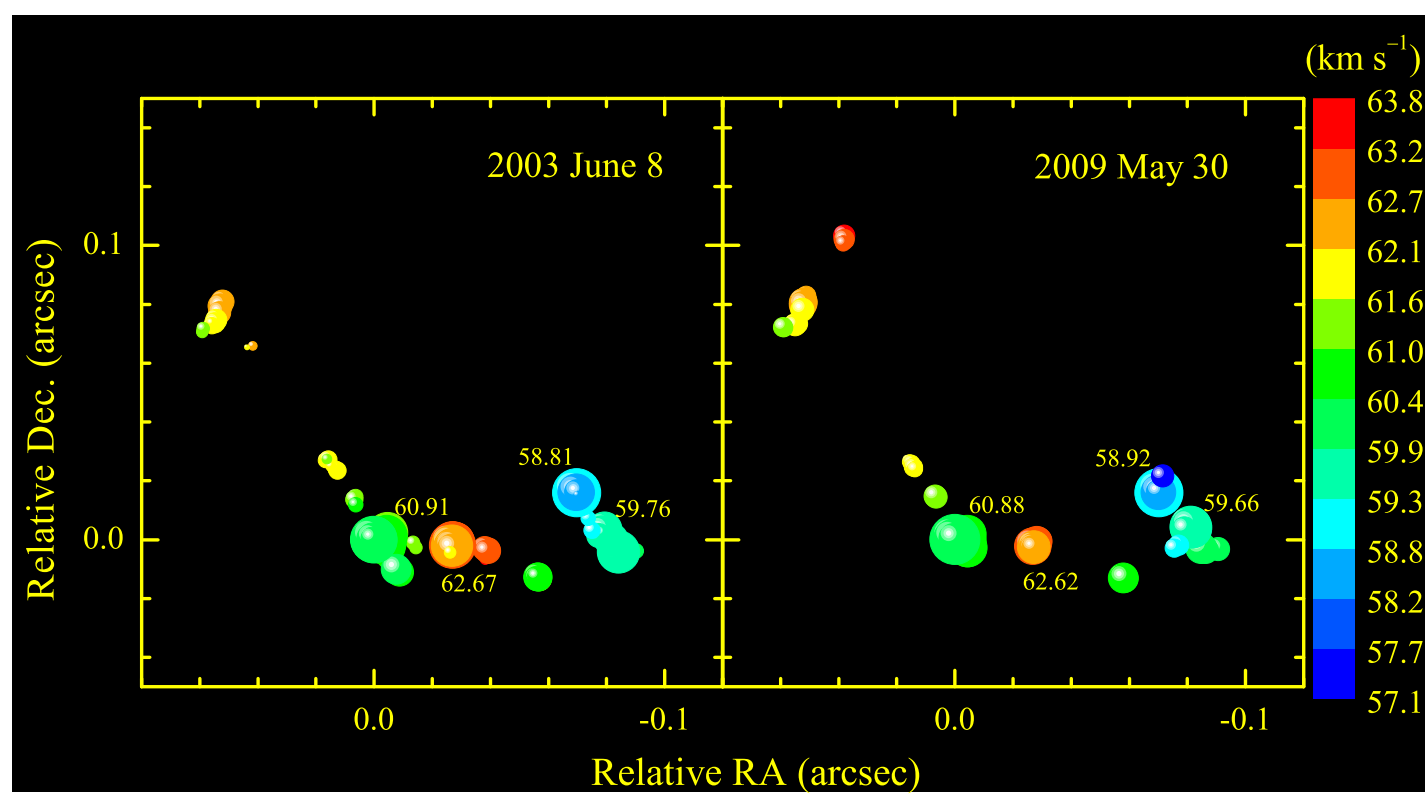

Figure 1: G33.641-0.228. EVN maps of $6.7 \mathrm{GHz}$ methanol maser emission at two epochs. The first epoch data are from [1]. The origin of maps is at $\mathrm{RA}(\mathrm{J} 2000)=18^{\mathrm{h}} 53^{\mathrm{m}} 32.5677, \operatorname{Dec}(\mathrm{J} 2000)=+00^{\circ} 31^{\prime} 39^{\prime \prime} 164$. The size of the symbols is proportional to the logarithm of brightness. The colour corresponds to the velocity scale shown by a vertical bar. Clusters of spots showing the velocity drifts are labelled by their central velocities at each epoch.

of $3.5 \mathrm{~km} \mathrm{~s}^{-1}$ for the assumed near-kinematic distance of $3.8 \mathrm{kpc}$. No regular internal motions of clusters were detected. There were the velocity shifts of 17.9 and $-17.5 \mathrm{~m} \mathrm{~s}^{-1} \mathrm{yr}^{-1}$ for clusters of the central velocity of 58.81 and $59.76 \mathrm{~km} \mathrm{~s}^{-1}$, respectively (Fig. 2). For three other clusters the velocity shifts were less than $7.5 \mathrm{~m} \mathrm{~s}^{-1} \mathrm{yr}^{-1}$. No obvious velocity gradient across the overall structure was seen. However, monotonic velocity gradients were found within individual clusters. These internal velocity gradients were almost similar at both epochs and did not show any regularity across the arc.

Comparison of the spectrum obtained at the second epoch with the single dish spectrum taken a month later implies that ratio $S_{\text {int }}(32 \mathrm{~m}) / S_{\text {int }}(E V N)$ is 0.58 , where $S_{\text {int }}(32 \mathrm{~m})$ and $S_{\text {int }}(E V N)$ are the integrated flux densities measured with the $32 \mathrm{~m}$ telescope and EVN, respectively. This means that about half of the maser flux density was missed in the EVN observations and implies a low brightness and extended emission which can be mapped with a lower angular resolution.

The target was monitored over $\sim 14$ months. Variations of the peak flux of clearly seen features are shown in Figure 2. A level of the variability was quantified as the ratio of maximum to minimum flux density and its time scale as the interval from minimum to maximum. Typically this ratio ranged from 1.2 to 2.6 but for features centered near 58.9 and $59.6 \mathrm{~km} \mathrm{~s}^{-1}$ was as high as 4.1 and 16.7 , respectively. The timescales of variability was $1-10$ months. A $59.6 \mathrm{~km} \mathrm{~s}^{-1}$ feature clearly showed a flare with a decay time of $\sim 30$ days. We conclude that individual features in the spectrum of G33.641-0.228 showed significant variations of the peak flux density within the time-span of our observations. Inspection of the spectrum taken in 1999 August reported in the discovery paper of that source indicates that the velocity range of maser emission was the same as in 2009 June but 

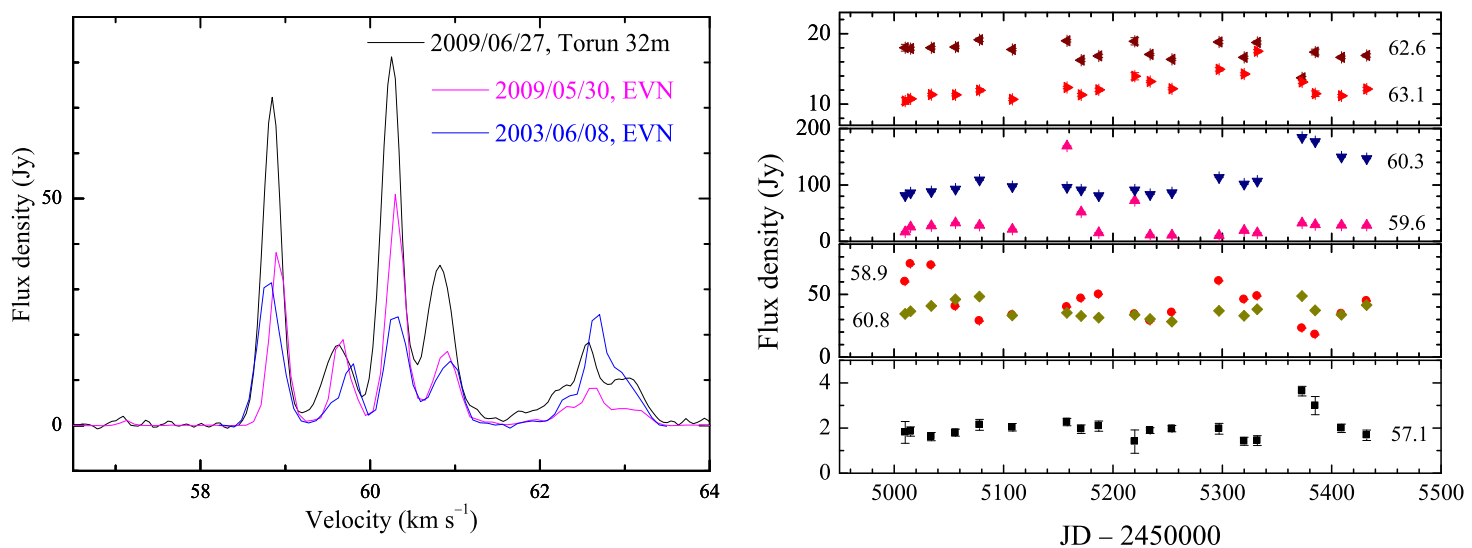

Figure 2: G33.641-0.228. Left: Spectra of 6.7 GHz maser line obtained with the Torun $32 \mathrm{~m}$ telescope and the EVN. Right: Time-series of the most prominent maser features labelled by their velocity in units $\mathrm{km} \mathrm{s}^{-1}$.

the shape was completely different.

The present study confirmed that source G33.641-0.228 is most variable object in our sample on time-scales of a few months to 6 years. The individual clusters distributed along the arc-like structure show noticeable variations including appearance and disappearance of groups of spots but the overall structure of the arc remains quite stable on time-scale of 6 years. A lack of any regularity in the velocity of the whole structure seems to exclude a scenario of a rotating disc. Velocity drifts detected for some clusters if interpreted as due to rotation, acceleration or deceleration of masing gas suggest a very modest motion. This seems to be consistent with the upper limit of $3.5 \mathrm{~km} \mathrm{~s}^{-1}$ for the relative tangential velocity. Its mean value is probably much lower but sufficient enough to change the maser path length resulting in variations of the maser flux. The maser is closely associated with a mid-IR object that has an excess of $4.5 \mu \mathrm{m}$ emission [1] which is evidence for shocked regions. We suggest that the maser arc outlines a shock front or a part of gas in outflow.

\section{References}

[1] Bartkiewicz, A., Szymczak, M., van Langevelde, H.J., Richards, A.M.S., \& Pihlström, Y.M., The diversity of methanol maser morphologies from VLBI observations, 2009, A\&A, 502, 155

[2] Caswell, J.L., Vaile, R.A.,\& Ellingsen, S.P. Variability of methanol masers, 1995, PASA, 12, 37

[3] Dodson, R., Ojha, R., \& Ellingsen, S.P., High-resolution observations of 6.7-GHz methanol masers with the Long Baseline Array, 2004, MNRAS, 351, 779

[4] Goedhart, S., Gaylard, M.J., \& van der Walt, D.J., Long-term monitoring of 6.7-GHz methanol masers, 2004, MNRAS, 355, 553

[5] Ott, M., Witzel, A., Quirrenbach, A., et al. An updated list of radio flux density calibrators, 1994, A\&A, 284, 331

[6] Minier, V., Booth, R.S., \& Conway, J.E. VLBI observations of 6.7 and $12.2 \mathrm{GHz}$ methanol masers toward high mass star-forming regions, 2000, A\&A, 362, 1093 